\title{
An aggressive and multi-pronged approach to prevent amputations in India
}

\author{
Vijay Viswanathan
}

Received: 13 August 2014 / Accepted: 18 August 2014 / Published online: 11 October 2014

(C) Research Society for Study of Diabetes in India 2014

Foot complications are a major cause of hospitalization in diabetes. Diabetic foot ulceration results from decreased blood supply, poor weight-bearing, loss of sensation and poor foot care. Foot complications both increase the health care costs and pose a heavy socioeconomic burden.

A study from India reported that the cost of diabetes care for a patient with foot ulcers was more than 4 times higher (INR 19 020; US\$409) than that for a patient without foot ulcers (INR 4493; US\$97) [1]. In India, most of the patients undergo major or minor amputations due to the failure of antimicrobial therapy against infections. The causes of amputations may vary based on geographical variations. An earlier hospitalbased multi-centric study to determine the prevalence of diabetic foot in India reported that the prevalence of foot infection among diabetic patients was 6-11\% and that of amputation was $3 \%$ [2]. Another multicentric study involving 31 study sites across India reported that major amputations comprised about $29.1 \%$ and minor amputations $70.9 \%$ among diabetic patients with foot infection, and $82 \%$ of them had neuropathy and $35 \%$ had peripheral vascular disease. This study also observed that infection was a significant cause of amputation in approximately $90 \%$ of the study population [3].

The study of Durgad et al. [4] emphasizes the microbial etiology of diabetic foot ulcers and their susceptibility patterns. They studied 70 diabetics and isolated 147 organisms from wound swabs and pus aspirates.

V. Viswanathan $(\triangle)$

M.V. Hospital for Diabetes and Prof. M. Viswanathan Diabetes Research Centre, (WHO Collaborating Centre for Research,

Education and Training in Diabetes), Royapuram,

Chennai 600 013, Tamil Nadu, India

e-mail: drvijay@mvdiabetes.com
About $42 \%$ of the diabetic patients were in poor glycemic control and more than $50 \%$ of patients studied had more serious form of infection as indicated by Wagner's grade 3-5 lesions. Out of 70 diabetics, $73 \%$ of patients were infected by the polymicrobial infection and the microbiological analysis revealed the high proportion of gram negative bacilli (57 \%) compared to gram positive cocci $(27 \%)$. Among gram positive cocci, $23 \%$ of Staphylococcus aureus isolates and $17 \%$ of coagulase negative Staphylococcal isolates were found to be methicillin resistant. As to gram negative bacilli, $23 \%$ of isolate belonged to the Enterobacteriaceae family and were extended spectrum beta lactamase producers. The high prevalence of multidrug-resistant organisms (MDROs) in the clinical settings, increase the extent of hospitalization and cost of management. These data report the prevailing existence of MDROs in the tertiary clinical settings and the diabetic patient's vulnerability to infections. Vulnerability to infection is due to defective inflammatory response against pathogens. Apart from susceptibility, the diabetic patients have defective wound healing due to defective phagocytic clearance, increased oxidative stress which causes prolonged inflammatory state and down regulation of different growth factors, resulting defective angiogenesis [5]. Bagdade et al. [6] observed reduced leukocyte phagocytosis in poorly controlled diabetes patients, and the improvement of microbicidal rates was directly correlated with correction of hyperglycemia. Diabetic patients have poor tolerance towards infections, and the infection adversely affects diabetic control. This recurring cycle leads to the uncontrolled rise in glucose levels and defective immune response to infections.

The treatment strategy for diabetic foot problems is complicated and often requires the attention of many 
specialists. According to Infectious Diseases Society of America (IDSA) guidelines and key recommendations, an empirical antibiotic regimen should be executed based upon culture and susceptibility analysis [7]. Additional therapies include the use of antibiotic impregnated beads, application of negative pressure wound therapy and hyperbaric oxygen treatment [8-10]. In the study of Durgad et al. [4], the empiric antibiotic therapy administered to the patients failed to act against MDROs and lead to progressive infection of the lesions which was evidenced by grade 3-5 lesions. Delayed or improper treatment of these infections leads to gangrene and amputation. It is unfortunate that a major amputation was done in $42.8 \%$ of patients. Most of the amputations can be prevented and limbs rescued through an aggressive and multipronged treatment of antibiotics, debridement, revascularization, and staged wound closure. As mentioned by Durgad et al. [4], timely evaluation of patients with diabetic foot ulcers will help to determine the prevalent flora, their susceptibility profile and also, help to formulate the empiric antimicrobial therapy. Therefore, there is an urgent need to prevent diabetic foot infection and amputation by ensuring good glycemic control, preventive foot care and prompt treatment of trivial foot lesions in people with diabetes. Besides this, imparting an awareness of diabetes, foot care, and the availability of podiatry services to the public, especially in subjects with diabetes will help to achieve blooming outcomes in this context.
Conflict of interest None.

\section{References}

1. Satyavani K, Hemalatha K, Shabana T, Vijay V. The costs of treating long term diabetic complications in a developing country: A study from India. J Assoc Physicians India. 2013;61:102-9.

2. Viswanathan V, Thomas N, Tandon N, et al. Profile of diabetic foot complications and its associated complications-A multicentric study from India. J Assoc Physicians India. 2005;53:933-6.

3. Viswanathan V, Kumpatla S. Pattern and causes of amputation in diabetic patients-A multicentric study from India. J Assoc Physicians India. 2011;59:148-51.

4. Durgad S, Koticha A, Nataraj G, Deshpande A, Mehta P. Diabetic foot ulcers - where do we stand microbiologically?. Int J Diabetes Dev Ctries. 2013. doi 10.1007/s13410-013-0180-9.

5. Carmeliet P. Mechanisms of angiogenesis and arteriogenesis. Nat Med. 2000;6(4):389-95.

6. Bagdade JD, Root RK, Bulger RJ. Impaired leukocyte function in patients with poorly controlled diabetes. Diabetes. 1974;23:9-15.

7. Lipsky BA, Berendt AR, Deery HG, Embil JM, Joseph WS, Karchmer AW, et al. Infectious diseases society of america diagnosis and treatment of diabetic foot infections. Clin Infect Dis. 2004;39: 885-910.

8. Krause FG, de Vries G, Meakin C, Kalla TP, Younger AS. Outcome of transmetatarsal amputations in diabetics using antibiotic beads. Foot Ankle Int. 2009;30:486-93.

9. Armstrong DG, Lavery LA. Negative pressure wound therapy after partial diabetic foot amputation: a multicentre, randomised controlled trial. Lancet. 2005;366:1704-10.

10. Chen CE, Ko JY, Fong CY, Juhn RJ. Treatment of diabetic foot infection with hyperbaric oxygen therapy. Foot Ankle Surg. 2010;16:91-5. 